\title{
A Two-Phase Poisson Process Model and Its Application to Analysis of Cancer Mortality among A-bomb Survivors
}

\author{
Megu Ohtaki ${ }^{* 1}, \quad$ Tetsuji Tonda $^{2}$, Kazuyuki Aihara $^{3}$ \\ ${ }^{* 1}$ Department of Environmetrics and Biometrics, Research Institute for Radiation \\ Biology and Medicine, Hiroshima University, 1-2-3 Kasumi, Minami-ku, Hiroshima \\ 734-8551, Japan \\ ${ }^{2}$ Department of Management Information Systems, Faculty of Management and \\ Information Systems, Prefectural University of Hiroshima, 1-1-71 Ujina-Higashi, \\ Minami-Ku, Hiroshima 734-8558, Japan. \\ ${ }^{3}$ Institute of Industrial Science, The University of Tokyo, 4-6-1 Komaba Meguro-ku, \\ Tokyo 153-8505, Japan
}

\begin{abstract}
We consider a two-phase Poisson process model where only early successive transitions are assumed to be sensitive to exposure. In the case where intensity transitions are low, we derive analytically an approximate formula for the distribution of time to event for the excess hazard ratio $(E H R)$ due to a single point exposure. The formula for $E H R$ is a polynomial in exposure dose. Since the formula for $E H R$ contains no unknown parameters except for the number of total stages, number of exposure-sensitive stages, and a coefficient of exposure effect, it is applicable easily under a variety of situations where there exists a possible latency time from a single point exposure to occurrence of event. Based on the multistage hypothesis of cancer, we formulate a radiation carcinogenesis model in which only some early consecutive stages of the process are sensitive to exposure, whereas later stages are not affected. An illustrative analysis using the proposed model is given for cancer mortality among A-bomb survivors.
\end{abstract}

Key words: Excess hazard ratio, Latency of cancer, Multistage model, Poisson process, Radiation carcinogenesis, Single point exposure

\footnotetext{
${ }^{* 1}$ Corresponding author. Tel.: +81-82 257 5852; Fax: +81-82 256 7106, E-mail: ohtaki@hiroshima-u.ac.jp
} 


\section{Introduction}

Many chronic diseases can be characterized by stages and may be expressed mathematically by an irreversible point process. As for human cancer, Muller (1951) and Nordling (1953) proposed the idea of a multistage model to explain the observation that mortality rates increase proportionately to the fifth or sixth power of age. The quantitative consequences of this theory were derived by Stocks (1953). Armitage and Doll (1954) considered that cancer was the end result of the accumulation in a normal cell of a critical number $(k)$ of independent transitions through a series of intermediate states, assuming that the cancer was caused by exposure to carcinogens at a fairly constant rate throughout life, and that age is the same as the duration of carcinogenic exposure. For most cases of cancer 5 to 7 stages are indicated for the value of $k$ (Doll, 1971). The multistage model has been incredibly useful as a conceptual tool to understand the role of time in carcinogenesis (Day, 1983). From the aspect of biological science, Vogelstein et al. (1988) revealed that the process of colorectal cancer, for example, consists of several carcinogenic mutations of specific genes. Molecular biologists have reinterpreted the theory as the "Hallmarks of Cancer" (Hanahanand and Weinberg, 2000, 2011). Chiang (1979) proposed a multistage model based on a non-stationary Poisson process to describe a chronic disease process, and derived explicit formulas for the density and distribution functions of time to event. Ohtaki and his colleagues (Ohtaki, 1981; Ohtaki et al., 1985; Ohtaki and Niwa, 2001) developed a generalized Armitage-Doll model based on an approximation to Chiang's result. Applying this model, Doi et al. (2011) analyzed cohort data on former workers in a poisonous gas factory and clarified that lung cancer incidence decreased with age at exposure to sulfur mustard. Pierce and Vaeth (2003) showed that the effects of exposure shift the age scale instead of acting multiplicatively on cancer rates, and that those effects can be specified by age and dose. As for analyses of the effects of the radiation exposure, Pierce et al. (1996), Preston et al. (2004), Izumi and Ohtaki (2004, 2007), and Ozasa et al. (2012) performed detailed analyses of the data from the Life Span Study (LSS) cohort of A-bomb survivors using a model for a single point radiation exposure. These traditional multistage carcinogenesis models, however, do not explain the evidence that the incidence of many solid tumors among the A-bomb survivors requires more than 20 years of latency from the radiation exposure due to the Atomic bomb (Shimizu et al. 1991). Whittemore (1977) considered time and age dependence of cancer incidence resulting from various types of carcinogenic exposures, assuming that the exposure affects one of several changes necessary for malignant cell transformation. Based on these studies, we extend the generalized Armitage-Doll model with non-stationary transitions to cope with the problem of long latency.

In Section 2, we present the mathematical formulation of the point process model. In Section 3 , the hazard ratio of time to event due to a single point exposure is considered. In 
Section 4, we show an application to the analysis of mortality risk from solid cancer among A-bomb Survivors. In Section 5, some discussions on mathematical modeling of carcinogenesis and speculations on the results of our data analysis are given.

\section{Two phase Poisson process}

In this section, we modify the multistage model due to Chiang (1979) such that only some early consecutive stages of the process are sensitive to the exposure of interest, whereas later stages are not affected by that exposure. In other words, we assume that there exists a stage $u$ at which the transition becomes irreversibly insensitive to the exposure of interest (Figure 1).

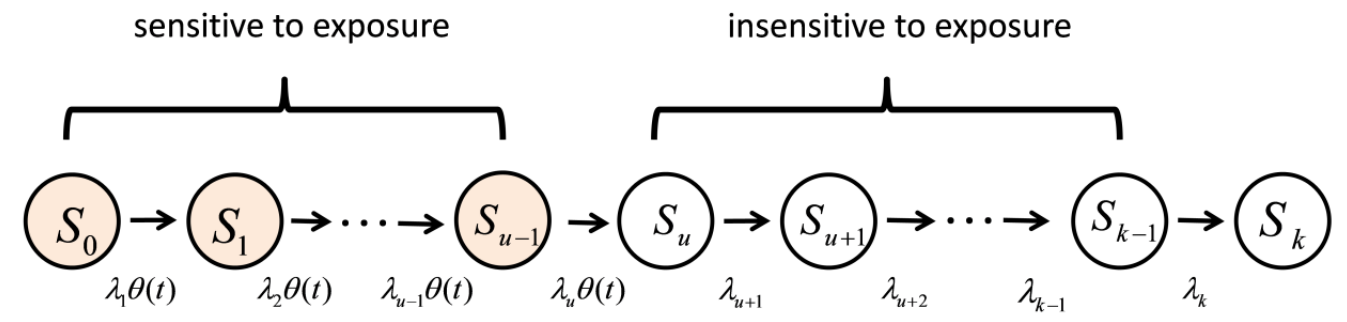

Figure 1. Schematic diagram of the two-phase irreversible $k$-stage point process $M(u, k-u)$

Definition 1. Suppose that there are $k$ stages in a process, $S_{0}, S_{1}, \ldots, S_{k-1}$ and a final stage $S_{k}$ and that the only possible transition in this process is $S_{j-1} \rightarrow S_{j}$ for $j=1,2, \ldots, k$. Given an element in stage $S_{j}$ at time $t$, the intensity of the transition $S_{j-1} \rightarrow S_{j}$ during the interval $(t, t+d t)$ is $\lambda_{j} \theta(t)$ if $j \leq u$ and $\lambda_{j}$ if $j \geq u+1, u=1, \ldots, k-1$ for all $0 \leq t<+\infty$, where $\lambda_{j}$ denotes a transition intensity at $S_{j-1} \rightarrow S_{j}$ in the case of non-exposure and $\theta(t)$ is the effect of exposure on the transition intensity at $t$. Let $T$ be the time at which an element that is initially in stage $S_{0}$ at time 0 enters the final stage $S_{k}$. We call this process the two-phase irreversible $k$ -stage point process, and denote it by $M(u, k-u)$ (see Figure 1).

As for the process $M(k-1,1)$, explicit formulas for the density and distribution functions of $T$ were given by Chiang (1979) and simple approximations with the gamma distribution were provided by Ohtaki (1981). We present them without 
detailed proof in the following proposition.

Proposition 1. Let $f_{k}(t \mid \lambda, \theta)$ and $F_{(k-1,1)}(t \mid \lambda, \theta)$ be the density and distribution

functions of $T$ in $M(k-1,1)$, respectively. Let $\Theta(t)=\int_{0}^{t} \theta(z) d z, t \geq 0$. If $\lambda_{i} \neq \lambda_{j}$, then for $i \neq j$,

$$
\begin{aligned}
& f_{(k-1,1)}(t \mid \lambda, \theta)=\lambda_{1} \cdots \lambda_{k} \theta(t) \sum_{i=1}^{k} \frac{1}{\prod_{\substack{j=1 \\
j \neq i}}^{k}\left(\lambda_{j}-\lambda_{i}\right)} \exp \left(-\lambda_{i} \Theta(t)\right), \\
& F_{(k-1,1)}(t \mid \lambda, \theta)=\lambda_{1} \cdots \lambda_{k} \sum_{i=1}^{k} \frac{1}{\prod_{\substack{j=1 \\
j \neq i}}^{k}\left(\lambda_{j}-\lambda_{i}\right) \lambda_{i}}\left\{1-\exp \left(-\lambda_{i} \Theta(t)\right)\right\}, \quad t \geq 0,
\end{aligned}
$$

and these are approximated by the following gamma-type density and distribution functions:

$$
\begin{aligned}
& g_{k}(t \mid \mu, \theta)=\frac{\mu^{k}}{(k-1) !} \theta(t)\{\Theta(t)\}^{k-1} \exp (-\mu \Theta(t)), \\
& G_{k}(t \mid \mu, \theta)=1-\exp (-\mu \Theta(t)) \cdot \sum_{j=1}^{k} \frac{\mu^{j-1}}{(j-1) !}\{\Theta(t)\}^{j-1},
\end{aligned}
$$

respectively, where $\mu=\sqrt[k]{\lambda_{1} \cdots \lambda_{k}}$. More precisely, we have

$$
\begin{aligned}
\sup _{0 \leq \tau \leq t} \frac{\left|f_{(k-1,1)}(\tau \mid \lambda, \theta)-g_{k}(\tau \mid \mu, \theta)\right|}{g_{k}(\tau \mid \mu, \theta)} \leq \sup _{0 \leq \tau \leq t} \frac{\left|F_{(k-1,1)}(\tau \mid \lambda, \theta)-G_{k}(\tau \mid \mu, \theta)\right|}{G_{k}(\tau \mid \mu, \theta)} \\
\leq \frac{1}{2}\{\exp ((\bar{\lambda}+\mu) \Theta(t))-\exp (2 \mu \Theta(t))+1-\exp (-(\bar{\lambda}-\mu) \Theta(t))\}, \quad t \geq 0,
\end{aligned}
$$

where $\bar{\lambda}=\max _{j=1, \ldots, k} \lambda_{j}$

Proof of Proposition 1:

Formulas (1) and (2) were derived by Chiang (1979; Theorem 2); the approximating formula and related inequalities were given in the theorem of Ohtaki (1981). 


\section{Excess hazard ratio due to single point exposure}

In this section we consider the excess relative hazard for arrival at the $k^{\text {th }}$ stage when a single point exposure exists. In the case of a single point exposure of dose $D$ at time $a$, the effect of the exposure on transition can be specified as

$$
\theta(t) \equiv \theta(t \mid D, a)=1+\beta_{a} D \cdot \delta(t-a),
$$

where $\beta_{a}$ is a function of $a$ and $\delta(t-a)$ is the Dirac delta function with support $a$. Then the intensity of the transition $S_{j-1} \rightarrow S_{j}$ during the interval $(t, t+d t)$ is

$\lambda_{j}\left\{1+\beta_{a} D \delta(t-a)\right\}$ if $j \leq u$ and $\lambda_{j}$ if $j \geq u+1$. As for the probabilities of occupying the various stages, we have the following lemma.

Lemma 1. Assume that $M(u, k-u)$ with a single point exposure of dose $D$ at time $a$ is given. Let $X_{t}$ be the stage number at time $t$ defined by $X_{t}=j$ if the stage is $j \in\{1,2, \ldots, k\}$. Denote $\lim _{\varepsilon \downarrow 0} \operatorname{Pr}\left(X_{a-\varepsilon}=j\right)$ and $\lim _{\varepsilon \downarrow 0} \operatorname{Pr}\left(X_{a+\varepsilon}=j\right)$ by $\operatorname{Pr}\left(X_{a-0}=j\right)$ and $\operatorname{Pr}\left(X_{a+0}=j\right)$, respectively. Then

$$
\begin{aligned}
& \operatorname{Pr}\left(X_{a-0}=j\right)=\frac{\lambda_{1} \cdots \lambda_{j}}{j !} a^{j} \cdot\left\{1+o\left(\max _{m=1, \ldots j} \lambda_{m}\right)\right\}, \\
& \operatorname{Pr}\left(X_{a+0}=j+\ell \mid X_{a-0}=j\right)=\frac{\lambda_{j+1} \cdots \lambda_{j+\ell}\left(\beta_{a} D\right)^{\ell}}{\ell !} \cdot\left\{1+o\left(\max _{m=j+1, \ldots, j+\ell} \lambda_{m}\right)\right\},
\end{aligned}
$$

and

$$
\operatorname{Pr}\left(X_{t}=k-1 \mid X_{a+0}=j+\ell\right)=\frac{\lambda_{j+\ell+1} \cdots \lambda_{k-1}}{(k-1-j-\ell) !}(t-a)^{k-1-j-\ell} \cdot\left\{1+o\left(\max _{m=j+\ell+1, \ldots k-1} \lambda_{m}\right)\right\},
$$

for $\ell=1, \ldots, u-j, j=1, \ldots, u$.

Proof of Lemma 1: Let $\mu_{j+1}=\sqrt[j+1]{\lambda_{1} \cdots \lambda_{j+1}}$, and

$$
\begin{aligned}
& f_{j+1}(t \mid \lambda)=\lambda_{1} \cdots \lambda_{j+1} \sum_{i=1}^{j+1} \frac{1}{\prod_{\substack{m=1 \\
m \neq i}}^{j+1}\left(\lambda_{m}-\lambda_{i}\right)} \exp \left(-\lambda_{i} t\right), \\
& g_{j+1}\left(t \mid \mu_{j+1}\right)=\frac{\left(\mu_{j+1}\right)^{j+1}}{j !} t^{j} \exp \left(-\mu_{j+1} t\right), \quad t \geq 0,
\end{aligned}
$$

for $j=1, \ldots, k-1$. Then, from (4) in Proposition 1 , 
where $\bar{\lambda}_{j+1}=\max _{i=1, \ldots, j+1} \lambda_{i}$. Therefore, we have

$$
f_{j+1}(t \mid \lambda)=g\left(t \mid \mu_{j+1}\right) \cdot\left\{1+o\left(\bar{\lambda}_{j+1}\right)\right\}=\frac{\lambda_{1} \cdots \lambda_{j+1}}{j !} t^{j} \cdot\left\{1+o\left(\bar{\lambda}_{j+1}\right)\right\} .
$$

Since $f_{j+1}(t \mid \lambda)=\lambda_{j+1} \cdot \operatorname{Pr}\left(X_{t}=j\right)$, for $0 \leq t<a$, Equation (6) follows from

$$
\operatorname{Pr}\left(X_{a-0}=j\right)=\lim _{t \uparrow a} \operatorname{Pr}\left(X_{t}=j\right)=\lim _{t \uparrow a} \frac{f_{j+1}(t \mid \lambda)}{\lambda_{j+1}}=\lim _{t \uparrow a} \frac{\lambda_{1} \cdots \lambda_{j}}{j !} t^{j} \cdot\left\{1+o\left(\bar{\lambda}_{j}\right)\right\} .
$$

Using similar arguments, we obtain (7) and (8).

Based on Lemma 1, we deduce the following theorem.

Theorem 1. Assume that $M(u, k-u)$ with single point exposure of dose $D$ at time $a$ is given. If $\lambda_{1}, \lambda_{2}, \ldots, \lambda_{k-1}$ are sufficiently small, then the excess hazard ratio at time $t$ can be expressed approximately as

$$
\begin{aligned}
& \operatorname{EHR}_{(u, k-u)}(t \mid D, a) \\
& \quad=\left(1-\frac{a}{t}\right)^{k-1-u} \sum_{\ell=1}^{u} \frac{(k-1) !}{\ell !(k-1-\ell) !}\left\{\sum_{j=0}^{u-\ell} \frac{(k-1-\ell) !}{j !(k-1-\ell-j) !}\left(\frac{a}{t}\right)^{j}\left(1-\frac{a}{t}\right)^{u-\ell-j}\right\}\left(\frac{\beta_{a} D}{t}\right)^{\ell},
\end{aligned}
$$

for $u=1, \ldots, k-1$.

Proof of Theorem 1:

Since

$$
\lim _{\Delta t \rightarrow 0} \frac{\operatorname{Pr}\left(X_{t+\Delta t}=k \mid X_{t}=k-1\right)}{\Delta t}=\lambda_{k},
$$

the hazard of the arrival to the $k^{\text {th }}$ stage at time $t$ can be expressed as

$$
h_{(u, k-u)}(t \mid D, a)=\lambda_{k} \cdot \operatorname{Pr}\left(X_{t}=k-1\right) \cdot\left\{1+o\left(\max _{j=1, \ldots k-1} \lambda_{j}\right)\right\} .
$$

Note that there are the following two cases of processes:

1) the stage at time (at exposure) $a$ is earlier than $u$, and

2) the stage at time $a$ is between $u$ and $k-1$,

which are disjoint. Then, it follows from (6), (7) and (8) in Lemma 1 that 


$$
\begin{aligned}
& \operatorname{Pr}\left(X_{t}=k-1\right) \\
& =\sum_{j=0}^{u-1} \sum_{\ell=0}^{u-j} \operatorname{Pr}\left(X_{a-0}=j\right) \cdot \operatorname{Pr}\left(X_{a+0}=j+\ell \mid X_{a-0}=j\right) \cdot \operatorname{Pr}\left(X_{t}=k-1 \mid X_{a+0}=j+\ell\right) \\
& \quad+\sum_{j=u}^{k-1} \operatorname{Pr}\left(X_{a-0}=j\right) \cdot \operatorname{Pr}\left(X_{a+0}=j \mid X_{a-0}=j\right) \cdot \operatorname{Pr}\left(X_{t}=k-1 \mid X_{a+0}=j\right) \\
& =\left\{\sum_{j=0}^{u-1} \sum_{\ell=0}^{u-j} \frac{\lambda_{1} \cdots \lambda_{j}}{j !} a^{j} \cdot \frac{\lambda_{j+1} \cdots \lambda_{j+\ell}}{\ell !}\left(\beta_{a} D\right)^{\ell} \cdot \frac{\lambda_{j+\ell+1} \cdots \lambda_{k-1}}{(k-1-j-\ell) !}(t-a)^{k-1-j-\ell}\right. \\
& \left.\quad+\sum_{j=u}^{k-1} \frac{\lambda_{1} \cdots \lambda_{j}}{j !} a^{j} \cdot \frac{\lambda_{j+1} \cdots \lambda_{k-1}}{(k-1-j) !}(t-a)^{k-1-j}\right\} \cdot\left\{1+o\left(\max _{j=1, \ldots, k-1} \lambda_{j}\right)\right\} \\
& =\frac{\lambda_{1} \cdots \lambda_{k-1}}{(k-1) !} t^{k-1} \cdot\left\{\sum_{j=0}^{u-1} \frac{(k-1) !}{j !(k-j-1) !}\left(\frac{a}{t}\right)^{j} \sum_{\ell=1}^{u-j} \frac{(k-1-j) !}{\ell !(k-1-j-\ell) !}\left(1-\frac{a}{t}\right)^{k-1-j-\ell}\left(\frac{\beta_{a} D}{t}\right)^{\ell}\right\} \\
& \cdot\left\{1+o\left(\max _{j=1, \ldots, k-1} \lambda_{j}\right)\right\} .
\end{aligned}
$$

Since the density function for the background hazard can be described as $h_{(u, k-u)}(t \mid 0, a)=\frac{\lambda_{1} \cdots \lambda_{k}}{(k-1) !} t^{k-1} \cdot\left\{1+o\left(\max _{j=1, \ldots, k-1} \lambda_{j}\right)\right\}$, it follows from (10) and (11) that the hazard ratio at time $t$ can be expressed as

$$
\begin{aligned}
& H R_{(u, k-u)}(t \mid D, a)=\frac{h_{(u, k-u)}(t \mid D, a)}{h_{(u, k-u)}(t \mid 0, a)} \cdot\left\{1+o\left(\max _{j=1, \ldots, k-1} \lambda_{j}\right)\right\} \\
& =\frac{\lambda_{k} \cdot \operatorname{Pr}\left(X_{t}=k-1 \mid D, a\right)}{\lambda_{1} \cdots \lambda_{k} t^{k-1} /(k-1) !} \cdot\left\{1+o\left(\max _{j=1, \ldots, k-1} \lambda_{j}\right)\right\} \\
& =1+\sum_{j=0}^{u-1} \frac{(k-1) !}{j !(k-j-1) !}\left(\frac{a}{t}\right)^{j} \sum_{\ell=1}^{u-j} \frac{(k-1-j) !}{\ell !(k-1-j-\ell) !}\left(1-\frac{a}{t}\right)^{k-1-j-\ell}\left(\frac{\beta_{a} D}{t}\right)^{\ell} \cdot\left\{1+o\left(\max _{j=1, \ldots k-1} \lambda_{j}\right)\right\},
\end{aligned}
$$

for $u=1, \ldots, k-1$. Hence, it follows from $\max _{j=1, \ldots, k-1} \lambda_{j} \doteq 0$ that

$$
\begin{aligned}
& \operatorname{EHR}_{(u, k-u)}(t \mid D, a) \equiv H R_{(u, k-u)}(t \mid D, a)-1 \\
& \quad \sum_{j=0}^{u-1} \frac{(k-1) !}{j !(k-j-1) !}\left(\frac{a}{t}\right)^{j} \sum_{\ell=1}^{u-j} \frac{(k-1-j) !}{\ell !(k-1-j-\ell) !}\left(1-\frac{a}{t}\right)^{k-1-j-\ell}\left(\frac{\beta_{a} D}{t}\right)^{\ell} \\
& \quad=\left(1-\frac{a}{t}\right)^{k-1-u} \sum_{\ell=1}^{u} \sum_{j=0}^{u-\ell} \frac{(k-1-\ell) !}{j ! \ell !(k-1-\ell-j) !}\left(\frac{a}{t}\right)^{j}\left(1-\frac{a}{t}\right)^{u-\ell-j}\left(\frac{\beta_{a} D}{t}\right)^{\ell},
\end{aligned}
$$

for $u=1, \ldots, k-1$. Thus, Theorem 1 is established.

It is noteworthy that Formula (9) for $E H R_{(u, k-u)}$ shows that the excess hazard 
ratio can be described by a $u^{\text {th }}$ order polynomial in dose $D$ which does not depend on the values of the transition parameters $\lambda_{1}, \ldots, \lambda_{k}$. When the exposure time $a$ is known, it contains no unknown parameters except for the number of total stages $k$, number of exposure-sensitive stages $u$, and coefficient of exposure effect $\beta_{a}$.

We then have the following obvious results:

$$
\begin{aligned}
& \operatorname{EHR}_{(1, k-1)}(t \mid D, a)=(k-1)\left(1-\frac{a}{t}\right)^{k-2} \frac{\beta_{a} D}{t}, \\
& \operatorname{EHR}_{(k-1,1)}(t \mid D, a)=\left(1+\frac{\beta_{a} D}{t}\right)^{k-1}-1,
\end{aligned}
$$

for $k=2,3, \ldots$. Formula (12) was also obtained by Pierce and Vaeth (2003).

Neglecting the quadratic and higher-order terms with respect to $\beta_{a} D / t$ in Formula (9) in Theorem 1, we obtain a "linear" approximation formula for $\operatorname{EHR}_{(u, k-u)}$ as follows:

$$
\operatorname{EHR}_{(u, k-u)}^{(1)}(t \mid D, a)=(k-1)\left(1-\frac{a}{t}\right)^{k-1-u}\left\{\sum_{j=0}^{u-1} \frac{(k-2) !}{j !(k-2-j) !}\left(\frac{a}{t}\right)^{j}\left(1-\frac{a}{t}\right)^{u-1-j}\right\} \frac{\beta_{a} D}{t},
$$

for $u=1, \ldots, k-1$, when $\beta_{a} D / t$ is sufficiently small.

\section{Application to analysis of cancer mortality among A-bomb Survivors}

In this section we apply the model to the analysis of cancer mortality among A-bomb Survivors. We assume that carcinogenesis is an irreversible multistage point process. The Radiation Effects Research Foundation (RERF) and its predecessor, the Atomic Bomb Casualty Commission (ABCC), have conducted a cohort study, called the Life Span Study (LSS), on A-bomb survivors in Hiroshima and Nagasaki since 1950. Ozasa et al. (2012) recently published the 14th report on cancer mortality as well as non-cancer mortality in the LSS, covering the period 1950-2003. The number of subjects analyzed was 86,611 (58,494 in Hiroshima and 28,117 in Nagasaki). The LSS data are available for public use on the RERF website (http://rerf.jp/).

We analyzed the data on solid cancer mortality using the two-phase multistage models $M(u, 6-u), u=1, \ldots, 5$, where the number of stages of carcinogenesis $k$ was assumed to be 6 based on Doll (1971). We then compared our results with those of Ozasa et al. (2012). The dose-response relationship for solid cancer incidence/mortality among A-bomb survivors in the LSS was considered to be 
linear rather than a higher order polynomial such as quadratic or cubic (e.g. Pierce et al., 1996, Ozasa et al., 2012). In keeping with their analysis, Poisson regression methods for grouped survival data with the linear approximation to the excess relative risk were used to describe the dependence of risk on radiation dose and to evaluate the variation of the dose response with respect to city, sex, age at exposure, and attained age. Analyses of all solid cancers were based on colon dose as representative of doses to all organs. The models we used are as follows.

For the excess relative risk $(E R R)$, which is equivalent to the excess hazard ratio $(E H R)$, a model of solid cancer death for an individual at age $t$ who was exposed to dose $D$ at age $a$ was assumed to be

$$
h(t \mid D, a, \operatorname{sex}, c)=h_{0}(t \mid a, \operatorname{sex}, c) \cdot[1+\operatorname{ERR}(t \mid D, a, \operatorname{sex})],
$$

where $h_{0}$ is the baseline or background mortality rate at zero (initial) dose, depending on city $(c)$, sex, birth year $(b \equiv 1945-a)$, and attained age $(t)$. The baseline mortality rate $h_{0}$ was modeled using stratification on city and birth year for the $E R R$ model. The $E R R$ model utilized at RERF is

$$
\operatorname{ERR}(t \mid D, a, \operatorname{sex})=(1+\sigma \operatorname{sex}) \cdot t^{v} e^{\tau a} \beta D,
$$

where the parameters $\tau, v$ and $\sigma$ are coefficients for effect modification by ATE (age at exposure), attained age, and sex, respectively. The term that includes sex ( $\operatorname{sex}=1$ for female and sex $=-1$ for male) as a modifier allows the parameter $\beta$ to represent a sex-averaged dose effect.

We obtain from (13) that the linear approximation to the excess relative risk of cancer mortality using the model $M(u, 6-u)$ can be specified as

$$
\begin{aligned}
& \operatorname{ERR}_{(u, 6-u)}^{(1)}(t \mid D, a, \operatorname{sex}) \\
& \quad=(1+\sigma \operatorname{sex}) \cdot\left(1-\frac{a}{t}\right)^{5-u}\left\{\sum_{j=0}^{u-1} \frac{120}{j !(4-j) !}\left(\frac{a}{t}\right)^{j}\left(1-\frac{a}{t}\right)^{u-1-j}\right\}\left(\frac{e^{\tau a} \beta D}{t}\right), u=1, \ldots, 5 .
\end{aligned}
$$

To implement these models, we used the generalized nonlinear model (gnm) package (Turner and Firth, 2007) in $\mathrm{R}$ (version 2.15.1). The resultant AIC, a measure of goodness of fit of the model (Akaike, 1973, 1992), is shown in Table 1. The minimum value of AIC was attained when the model of $\operatorname{ERR}_{(4,2)}^{(1)}$ based on $M(4,2)$ was used, which implies that some latency time is required from the time of exposure (due to the A-bomb radiation) to the actualization of the excess risk of death from cancer among subjects in the LSS; we call this our "optimal model". Figure 2 shows the estimated excess relative risk by 
age at exposure for a subject who was exposed to $1 \mathrm{~Gy}$, where the shaded curve indicates the projected $E R R$ for the period without direct follow-up in the LSS. Tables 2.1 and 2.2 show the estimated regression coefficients based on RERF's ERR model and our optimal model $E R R_{(4,2)}^{(1)}$ based on $M(4,2)$, respectively. The results of Table 2.1 coincide with those of Table 6 in Ozasa et al. (2012).

Compared with RERF's ERR model, although the estimated values of dose effect parameters $(\beta)$ and modifying effect of age at exposure on the dose effects $(\tau)$ were quit similar, there existed a large difference in the estimated trends of $E R R$ in relation to attained age after the bombing. Our proposed model suggested that no (or very low) ERR was expected during some period just after the bombing irrespective of age at exposure, and attained a peak with a slower rise to peak $E R R$ particularly at older ages at exposure. On the other hand, the expected ERR in RERF's ERR model attained a peak just after exposure and decreased monotonically thereafter. It should be noted that crude values of dose effect parameters in the two models have different meanings, because the way in which the dose effect is varying with attained age in our proposed model differs from that of RERF's model. Therefore, the comparison of dose effects between the two models should be modified by the aging effect, which corresponds to the aging trend of excess relative risks given in Figure 2.

As a theoretical aspect of our proposed model, the aging trend of excess cancer risk in $M(5,1)$ becomes a maximum at the time of ATE and decreases in inverse proportion to attained age. The aging trend of RERF's ERR is similar to that of $M(5,1)$, except for including the additional parameter $v$, in the RERF's model the estimate of $v$ for LSS data was -0.86 . In the proposed model with no latency time $M(5,1)$, the value of $v$ is specified automatically as -1 . We consider that this shrinkage is a consequence of the effect of latency time in radiation carcinogenesis. Therefore, the ERR model without a latency time is not suitable to describe the ageing trend in the dose effect. Although there is no latency time in $M(5,1)$ and RERF's model, $M(6-u, u), u=2, \ldots 5$, models reflect the effect of latency time based on the number of sensitive stage $u$. Table 1 shows AIC of candidate models for the LSS data, which provides basic information on the numerical comparison of performance between RERF's model and $M(6-u, u)$, $u=1,2, \ldots, 5$. The improved performance of our optimal model using fewer free parameters compared with that of the traditional model, which is indicated by a reduction of 2.39 in AIC, may not be astounding (see Table 1, Table 2.1 and Table 2.2). The minor difference in their performance may be due to lack of information about cancer mortality among survivors in the early years just after the bombing. it was also noted that about $10 \%$ higher value was estimated 
for the coefficient of dose effect, and that about $23 \%$ smaller value was estimated for the modifying effect of age at exposure on the dose effect in our model. Note that the main differences of aging trends of ERR in RERF's model and $M(6-u, u), u=1,2, \ldots, 5$ appear in the initial period after the bombing.

Table 1. Goodness of fit (AIC) of candidate models for the LSS data: the numerical comparison of performance between RERF's model and $M(6-u, u), u=1,2, \ldots 5$

\begin{tabular}{|c|c|}
\hline Model & AIC \\
\hline RERF's ERR model & 33838.36 \\
\hline$E R R_{(5,1)}^{(1)}$ model based on $M(5,1)$ & 33836.50 \\
\hline$E R R_{(4,2)}^{(1)}$ model based on $M(4,2)$ & $\mathbf{3 3 8 3 5 . 9 7}$ \\
\hline $\operatorname{ERR}_{(3,3)}^{(1)}$ model based on $M(3,3)$ & 33838.43 \\
\hline $\operatorname{ERR}_{(2,4)}^{(1)}$ model based on $M(2,4)$ & 33846.92 \\
\hline $\operatorname{ERR}_{(1,5)}^{(1)}$ model based on $M(1,5)$ & 33859.61 \\
\hline
\end{tabular}

Table2.1 Estimated regression parameters for RERF's ERR model

\begin{tabular}{|l|c|c|c|c|}
\hline \multicolumn{1}{|c|}{ Parameter } & Coef. & s.e & z-value & p-value \\
\hline$\beta$ : dose effect & 0.4226 & 0.0503 & 8.397 & $<0.001$ \\
\hline$\sigma:$ sex (female =1, male =-1) & 0.3441 & 0.0878 & 3.918 & $<0.001$ \\
\hline$\tau:$ age at exposure (per 10-year increment) & -0.3462 & 0.0814 & -4.254 & $<0.001$ \\
\hline$\nu:$ attained age (log(age 70$))$ & -0.8576 & 0.4232 & -2.026 & 0.0427 \\
\hline
\end{tabular}

Table 2.2 Estimated regression parameters based on our optimal model $E R R_{(4,2)}^{(1)}$

\begin{tabular}{|l|c|c|c|c|}
\hline \multicolumn{1}{|c|}{ Parameter } & Coef. & s.e & z-value & p-value \\
\hline$\beta:$ dose effect & 0.4609 & 0.0532 & 8.664 & $<0.001$ \\
\hline$\sigma:$ sex (female $=1$, male $=-1$ ) & 0.3375 & 0.0878 & 3.846 & $<0.001$ \\
\hline$\tau:$ age at exposure (per 10-year increment) & -0.2824 & 0.0752 & -3.758 & $<0.001$ \\
\hline
\end{tabular}



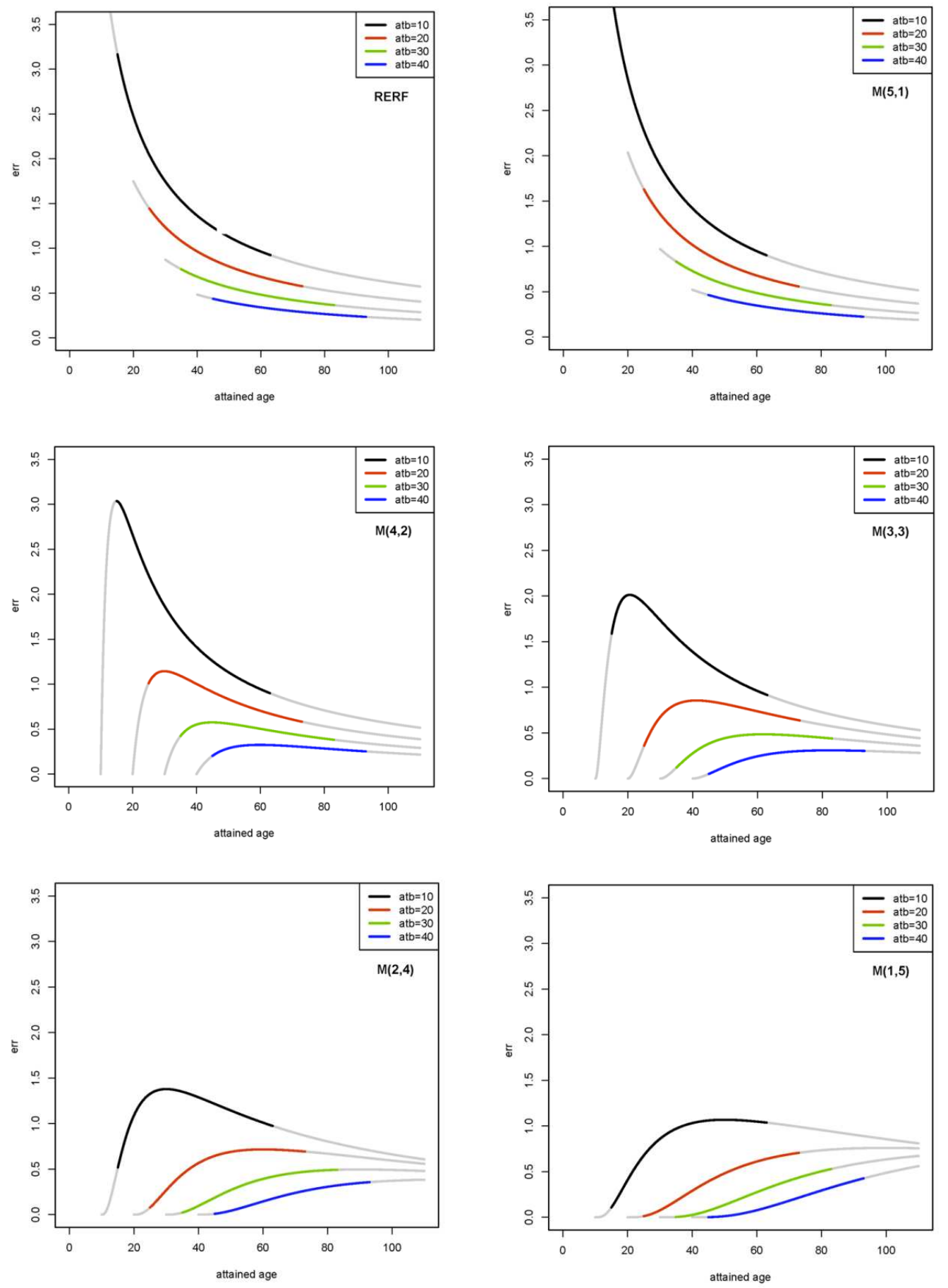

Figure 2. Theoretical curves of $E R R / G y$ of solid cancer mortality among subjects in the LSS by age at exposure and attained age obtained from the candidate models 


\section{Discussion:}

Our proposed multistage model of carcinogenesis accommodates fundamentally only single events such as cellular mutations. However, the last event in the process should be related more closely to tumor growth than to cellular transformation. A more explicit model for the carcinogenesis process may require that a mechanism of clonal expansion be incorporated in the model, such as with the two-stage clonal expansion (TSCE) model of Moolgavkar and Venzon (1979), which assumes clonal expansion as a stochastic process after two exposure-sensitive stages of cellular change in the earliest part of carcinogenesis. The TSCE model consists of not only transition parameters related to cellular stage as in the ordinary multistage model but also parameters concerning the clonal expansion (birth and death) process. To analyze the dose-response relationship, it is necessary to use a model having a structure for describing the dose effect. In the case of a single point exposure like radiation exposure due to the atomic bomb, a structure common to that of the ordinary multistage model for the cellular transition parameter may be assumed in the TSCE model (Kai et al., 1997). However, it is difficult to formulate the effect on the birth and death process, in that it is not easy to formulate the exposure effect on ERR in relation to attained age after exposure; therefore, we have not compared its performance with our model in actual data analysis. As for quantification of carcinogenesis, there are more recent models that do not fall within the Armitage-Doll or TSCE frameworks, which are regarded as extensions of the TSCE model having more complex structures with many additional parameters (Little, 1995; Aalen and Tretli, 1999; Luebeck and Moolgavkar, 2002; Nowak et al., 2007; Little and Wright, 2003; Beerenwinkel et al., 2007; Meza et al., 2008).

For illustrative application of the proposed model, we think that use of incidence data rather than mortality data is more reasonable, and would clearly provide a better test of the fit of our proposed model. However, the downloadable incidence data is limited to only observations taken from the period 1958 to 1998, while the latest version of mortality data which is available for us covered the wider period from 1950 to 2003. Use of the data of the early period around 1950 (just after the A-bomb exposure) is very important to clarify the performance of our proposed model. Therefore, we chose the mortality data rather than incidence data. We know the demerit of the use of mortality data for the test of the fit of our model. Actually, the length of clinical period of cancer (the period from cancer diagnosis to death, which has been assumed about several years) is ignored in our model. The last transition to the final stage may mean the clinical period, with some lack of accuracy, in our carcinogenesis model based on the Poisson process.

The result of our analysis of cancer mortality data among A-bomb survivors 
suggests a perspective "ionizing radiation works mainly as initiation" so that radio-therapy has been commonly applied to cancer patients. If ionizing radiation operates positively at late stages in carcinogenesis, cancer risk among exposed people would begin to increase soon after exposure, which was not observed in epidemiological studies (e.g., Shimuzi, et al., 1991; Travis, 2006). It was shown that a model with later stages of carcinogenesis insensitive to radiation fit the LSS solid cancer mortality data quite well, which provides a mathematical explanation for the latency time of appearance of the excess cancer risk among A-bomb survivors after radiation exposure. We think that the reason why the later stages of carcinogenesis are insensitive to radiation can be explained by the difference of target size for carcinogenic change among stages and the effect of radiation-induced apoptosis (Dewey et al., 1995). In the earlier stages, not only exons including oncogenes or suppressor genes but also introns are supposed to be the target (Nomura, 1982; Ohtaki and Niwa, 2001). On the other hand, in the later stages a few specific genes such as src, ras, and myc can be the target, where carcinogenic transitions should be rate-limiting events. Thus, the earlier stages have a relatively large target whereas the later stages have a small one. It is also well known that most irradiated cells are more susceptible to apoptosis than to carcinogenic transformation, which must reduce the sensitivity to radiation-induced carcinogenic transition in cells at later stages. With other kinds of exposure, such as chemicals, no latency time may exist in the carcinogenic process where all stages of cell transformation must be sensitive to exposure. For example, no latency time was expected between mustard gas exposure and excess risk of lung cancer morbidity among workers in the Ohkunojima Poisonous-gas factory in Japan (Doi et al., 2011).

\section{Acknowledgements}

This research was supported by the Ministry of Education, Culture, Sports, Science and Technology, Scientific Research (A) \#24249039 (2012-2014), and by the Aihara Project, the FIRST program from JSPS, initiated by CSTP, and CREST, JST.

This report makes use of data obtained from the Radiation Effects Research Foundation (RERF), Hiroshima and Nagasaki, Japan. RERF is a private, non-profit foundation funded by the Japanese Ministry of Health, Labor and Welfare (MHLW) and the U.S. Department of Energy (DOE), the latter in part through DOE Award DE-HS0000031 to the National Academy of Sciences. The conclusions in this report are those of the authors and do not necessarily reflect the scientific judgment of RERF or its funding agencies. The authors would like to thank the editor and referees for their constructive comments on the earlier version of this paper.

Conflict of interest: none declared. 


\section{References}

Akaike, H. (1973). Information theory and an extension of the maximum likelihood principle", Proceedings of the 2nd International Symposium on Information Theory, Petrov, B. N., and Caski, F. (eds.), Akadémiai Kiadó, Budapest: 267-281.

Akaike, H.(1992). Information theory and an extension of the maximum likelihood principle, "Breakthroughs in Statistics, Springer Series in Statistics1992, pp 610-624.

Aalen, O.O. and Tretli, S. (1999). Analyzing incidence of testis cancer by means of a frailty model. Cancer Causes Control 10, 285-289.,

Armitage, P. and Doll, R. (1954). The age distribution of cancer and a multi-stage theory of carcinogenesis. British Journal of Cancer 8, 1-12.

Beerenwinkel, N., Antal, T., Dingli, D., Traulsen, A., Kinzler, K.W., Velculescu, V., Vogelstein, B., and Nowak, M. (2007). Genetic progression and the waiting time to cancer. PLos Comput Biol 3(11): e225. doi: 10.1371/journal.pcbi.0030225.

Chiang, C.L. (1979). Survival and stages of disease, Mathematical Biosciences 43, 159-171.

Day, N. (1983). Time as a determinant of risk in cancer epidemiology: the role of multi-stage models. Cancer Surveys, 2:577-93, 1983.

Dewey,W.C., Ling,C.C. and Meyn, R.E. (1995). Radiation-induced apoptosis: relevance to radiotherapy. International Journal of Radiation Oncology • Biology · Physics, 33(4):781-96.

Doi, M., Hattori, N., Yokoyama, A., Onari, Y., Kanehara, M., Masuda, K., Tonda, T., Ohtaki M., and Kohno, N (2011). Effect of mustard gas exposure on incidence of lung cancer: a longitudinal study. American Journal Epidemiology 173, 659-666. doi: 10.1093/aje/kwq426.

Hanahan, D. and Weinberg, R. (2000). The hallmarks of cancer. Cell, 100: 57-70.

Hanahan, D. and Weinberg, R. (2011). Hallmarks of cancer: the next generation. Cell, 144(5): 646-74.

Izumi, S. and Ohtaki, M. (2004). Aspects of the Armitage-Doll gamma frailty model for cancer incidence data. Environmetrics 15, 209-218.

Izumi, S. and Ohtaki, M. (2007). Incorporation of inter-individual heterogeneity into the multistage carcinogenesis model: approach to the analysis of cancer incidence data. Biometrical Journal $\mathbf{4 9}$, 539-550. doi: 10.1002/bimj.200510336.

Kai, M., Luebeck, E.G. and Moolgavkar, S.H. (1997). Analysis of the incidence of solid cancer among atomic bomb survivors using a two-stage model of carcinogenesis. Radiation Research 148, 348-358.

Little, M.P. (1995). Are two mutations sufficient to cause cancer? Some generalizations of the two-mutation model of carcinogenesis of Moolgavkar, Venzon, and Knudson, and of the multistage model of Armitage and Doll. Biometrics 51, 1278-1291. 
Little, M.P. and Wright, E.G. (2003). A stochastic carcinogenesis model incorporating genetic instability fitted to colon cancer data. Mathemical Biosciences 183, 111-134.

Luebeck, E. G. and Moolgavkar, S.H. (2002). Multistage carcinogenesis and the incidence of colorectal cancer, Proceedings of the National Academy of Sciences of the United States of America 99, 15095-15100.

Meza, R., Jeon, J., Suresh H. Moolgavkar, S.H. and E. Georg Luebeck, E.G. (2008). Age-specific incidence of cancer: Phases, transitions, and biological implications. Proceedings of National Academy of Sciences 105, 16284-16289.

Moolgavkar, S.H. and Venzon, D.J. (1979). Two-event models for carcinogenesis: Incidence curves for childhood and adult tumors. Mathematical Biosciences 47, 55-77.

Muller, H.J. (1951). Radiation damage to the genetic material. Science in Progress 7, 93-177.

Nomura, T. (1982). Parental exposure to X-rays and chemicals induces heritable tumors and anomalies in mice. Nature 296, 575-577.

Nordling, C.O. (1953). A new theory on the cancer-inducing mechanism. British Journal of Cancer 7, 68-72.

Ohtaki, M. (1981). An approximation of the left tail of a distribution for waiting-time in an irreversible point process. Journal of the Japan Statistical Society 11, 111-118.

Ohtaki, M., Fujita, S., Hayakawa, N., Kurihara, M., and Munaka, M. (1985). The age distribution of human adult cancer and an initiation-manifestation model for carcinogenesis. Japanese Journal of Clinical Oncology 15, Suppl 1:325-343.

Ohtaki, M. and Niwa, O. (2001). A mathematical model of radiation carcinogenesis with induction of genomic instability and cell death. Radiation Research 156, 672-677.

Ozasa, K., Shimizu, Y., Suyama, A., Kasagi, F., Soda, M., Grant, E.J., Sakata, R., Sugiyama, H. and Kodama, K. (2012). Studies of the mortality of atomic bomb survivors, Report 14, 1950-2003: An overview of cancer and noncancer diseases. Radiation Research 177, 229-243.

Pierce, D.A., Shimizu, Y., Preston, D.L., Vaeth, M. and Mabuchi, K. (1996). Studies of the mortality of atomic bomb survivors. Report 12, Part 1. Cancer: 1950-1990. Radiation Research 146, $1-$ 27.

Pierce, D.A. and Vaeth, M. (2003). Age-time patterns of cancer to be anticipated from exposure to general mutagens. Biostatistics 4, 231-248.

Preston, D.L., Pierce, D.A., Shimizu, Y., Cillings, H.M., Fujita, S., Funamoto, S. and Kodama, K. (2004). Effect of recent changes in atomic bomb survivor dosimetry on cancer mortality risk estimates. Radiation Research 162, 377-389.

Shimizu, Y., Kato, H. and Schull, W.J (1991): Risk of cancer among atomic bomb survivors, Journal of Radiation Research., Supplement 2, 54-63. 
Stocks, P. (1953). A study of the age curve for cancer of the stomach in connection with a theory of the cancer producing mechanism. British Journal of Cancer 7, 407-417.

Travis L.B. (2006). The epidemiology of second primary cancers. Cancer Epidemiology, Biomarkers and Prevention 15, 2020-2026. doi: 10.1158/1055-9965.EPI-06-0414.

Turner, H. and Firth, D. (2007). gnm: A Package for Generalized Nonlinear Models. R News. 7, 8-12.

Vogelstein, B., Fearon, E.R., Hamilton, S.R.., Kern, S.E., Preisinger, A.C., Leppert, M., Nakamura, Y., White, R., Smits, A.M. and Bos, J.L. (1988). Genetic alterations during colorectal-tumor development. New England Journal of Medicine 319, 525-532.

Whittemore, A.S. (1977). The age distribution of human cancer for carcinogenic exposures of varying intensity, American Journal of Epidemiology 106, 418-432. 